\title{
PROVISION OF DIABETES CARE IN PRIMARY HEALTH \\ CARE CENTER IN THE UNITED ARAB EMIRATES
}

\section{INTRODUCTION}

Non communicable diseases have evolved as a public health concern and are one of the leading causes of mortality in the United Arab Emirates. Data also indicate that the prevalence of diabetes in the UAE is rising and expected to double to 2.2 million by 2040. The UAE national agenda 2021 is a turning point for scaling up diabetes care at primary care level. The national agenda 2021 includes a set of long-term indicators, focusing on national priorities including diabetes care aiming to reduce diabetes prevalence and mortality $30 \%$ by the year 2021. Building on the vital role of primary health care in diabetes screening and management, Ministry of Health and Prevention, launched its initiative to integrate NCD screening and management into PHC across UAE. The initiative focused on improving diabetes care using the WHO/ISH risk prediction chart and national diabetes guideline for screening and effective management.

\section{THE AIMS}

This study aims to assess implementation of the total cardiovascular (CVD) isk approach and diabetes national guideline, determining enabling factors that should accompany Ministry of health and prevention's attempt to expand coverage.
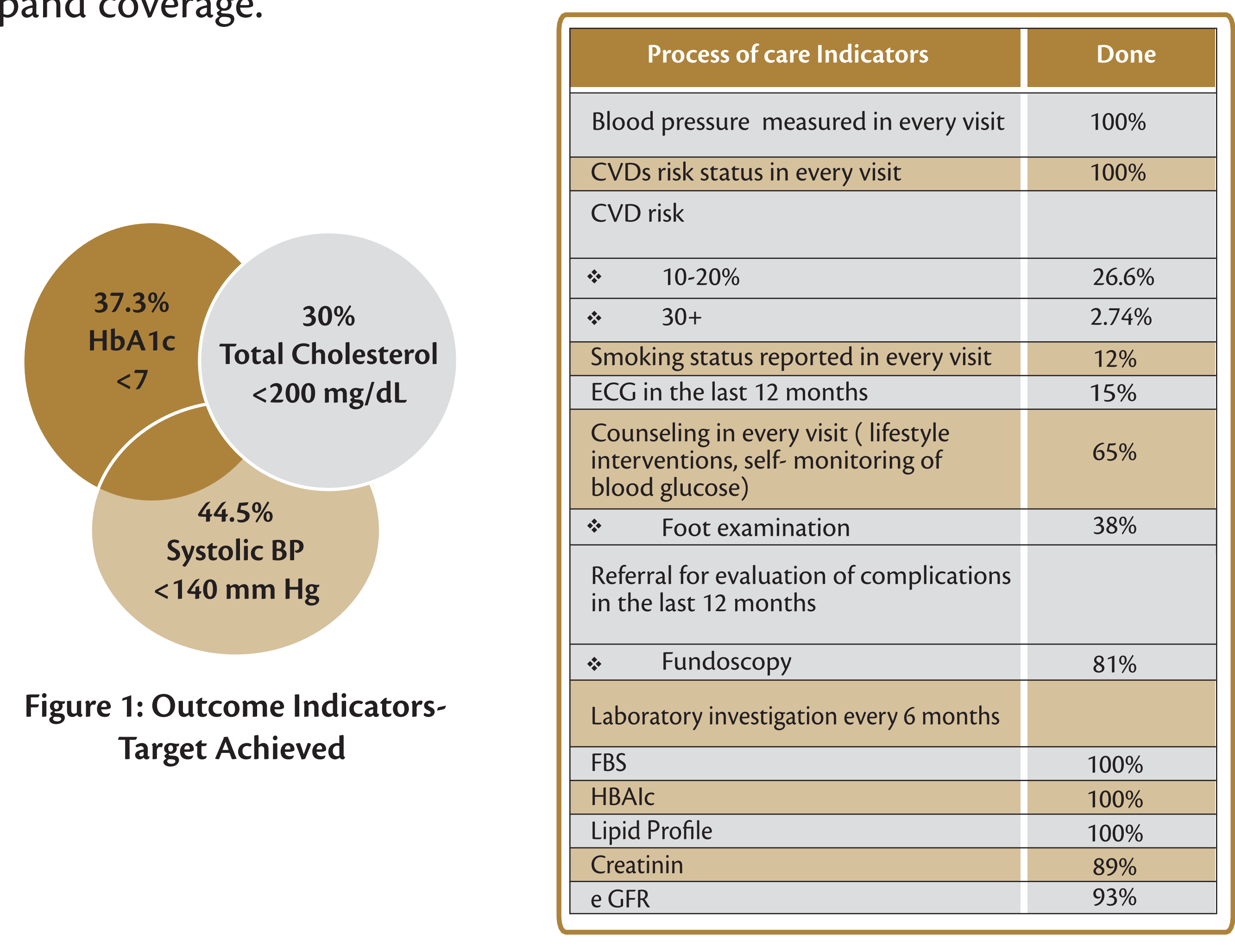

Table 1: Evaluation of process measures

\section{METHODS}

In total 6089 Diabetic patient records attending the Non communicable diseases clinics in the primary health care, UAE were reviewed from January 2016 to July 2017. Patient data was collected from patient's diabetes flow-chart, and performance measures of diabetes care were reviewed and reported. Data includes, duration of diabetes, associated comorbidity, CVDs risk score clinical measurement at each visit ECC. Evaluation of patients for clinical complications (referral), ie, eye examination, foot risk assessments, as well as, counseling (lifestyle intervention, self-monitoring of blood glucose) were reviewed and reported. In addition, selected outcome indicators such as (targeted HBAIc, targeted BP, and lipid profile) were analyzed through SPSS.
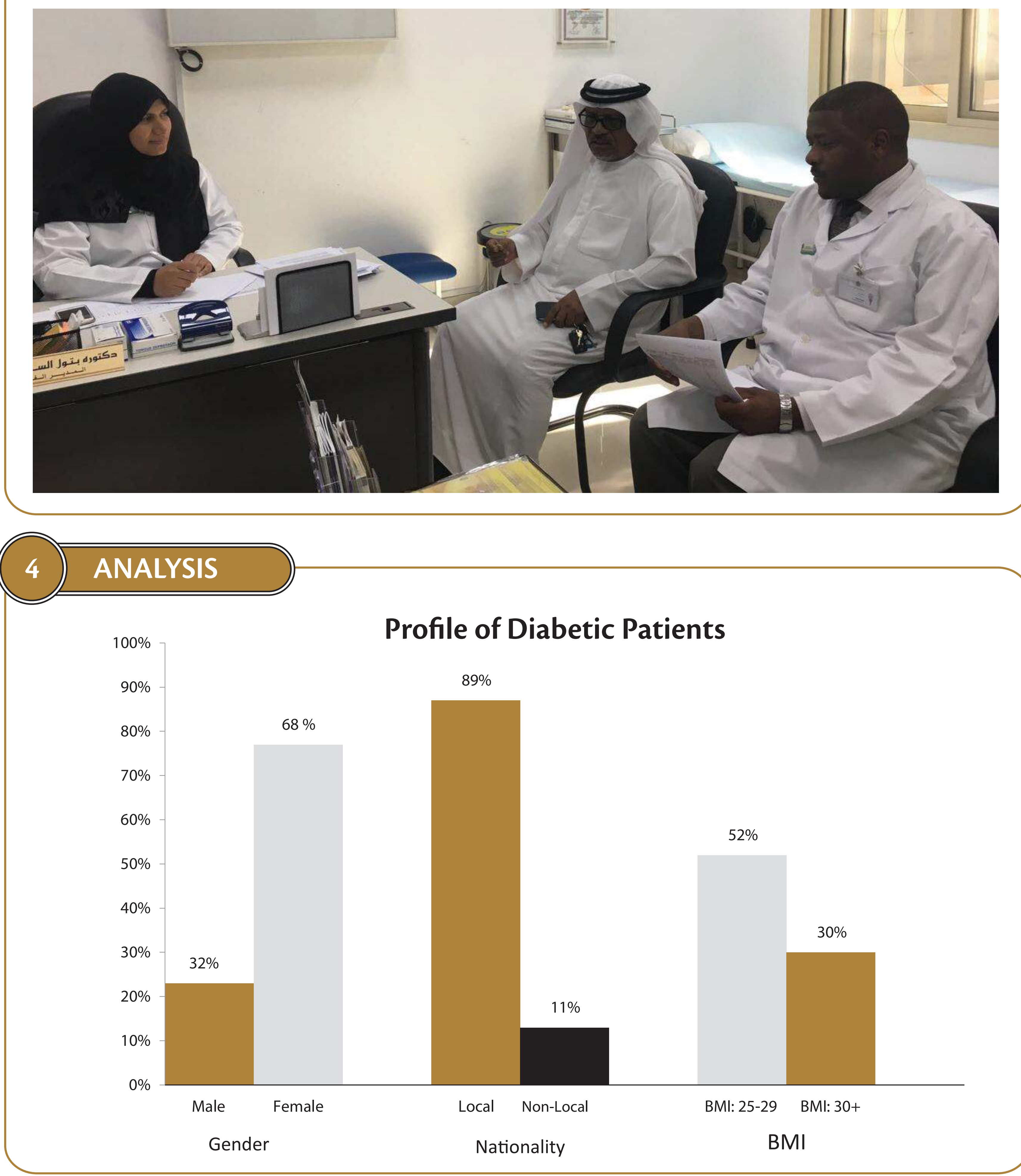

Presented by :Dr. Buthaina Abdullah Bin Belaila

\section{FINDINGS}

A total of 12296 diabetic patient records were reviewed. The average age was 58.9 years, majority (68\%) were females, and $89 \%$ were locals. Comorbidity (hypertension) was present in $43.3 \%$, and $82 \%$ were either obese $(30 \%$ ) or overweight (52\%). Evaluation of the intervention process reported gaps in few of the management processes (i.e. only $15 \%$ were referred for ECG during the last year, $81 \%$ were referred for Fundoscopy, and $38 \%$ for Foot examination). Furthermore, only $12 \%$ were enquired about smoking status. Overall, $65 \%$ were given counseling interventions at each vist. Evaluation of the CVD risk score indicated that $26.6 \%$ of patients had their 10 - years cardiovascular risk between $10-20 \%$ while only $2.74 \%$ have a 10 -year cardiovascular risk $>30 \%$. Figure 1 demonstrated that good diabetes control was achieved among $37.3 \%$ (with HbA1c $<7$ ), Blood pressure $(<140 \mathrm{mmHg}$ ) was controlled among $44.5 \%$ of the patients, while total Cholesterol $(<200 \mathrm{mg} / \mathrm{dL})$ was controlled among $30 \%$ of patients.

\section{CONCLUSION}

The audit confirmed that PHC providers follow national diabetes guideline for diabetes and hypertension with limitation. While introduction of CVDs risk stratification at primary health care proved to be a useful tool to enhance quality of management, yet the counseling skills require further improvement. Integrating clinical auditing into PHC and training of health providers should be a priority to scale up management of diabetes. There is a need to empower diabetic patients to improve outcome and adherence. Additional studies are needed in future to understand the long term impact of this strategy so as to improve the quality of Type 2 Diabetes Mellitus care across UAE.

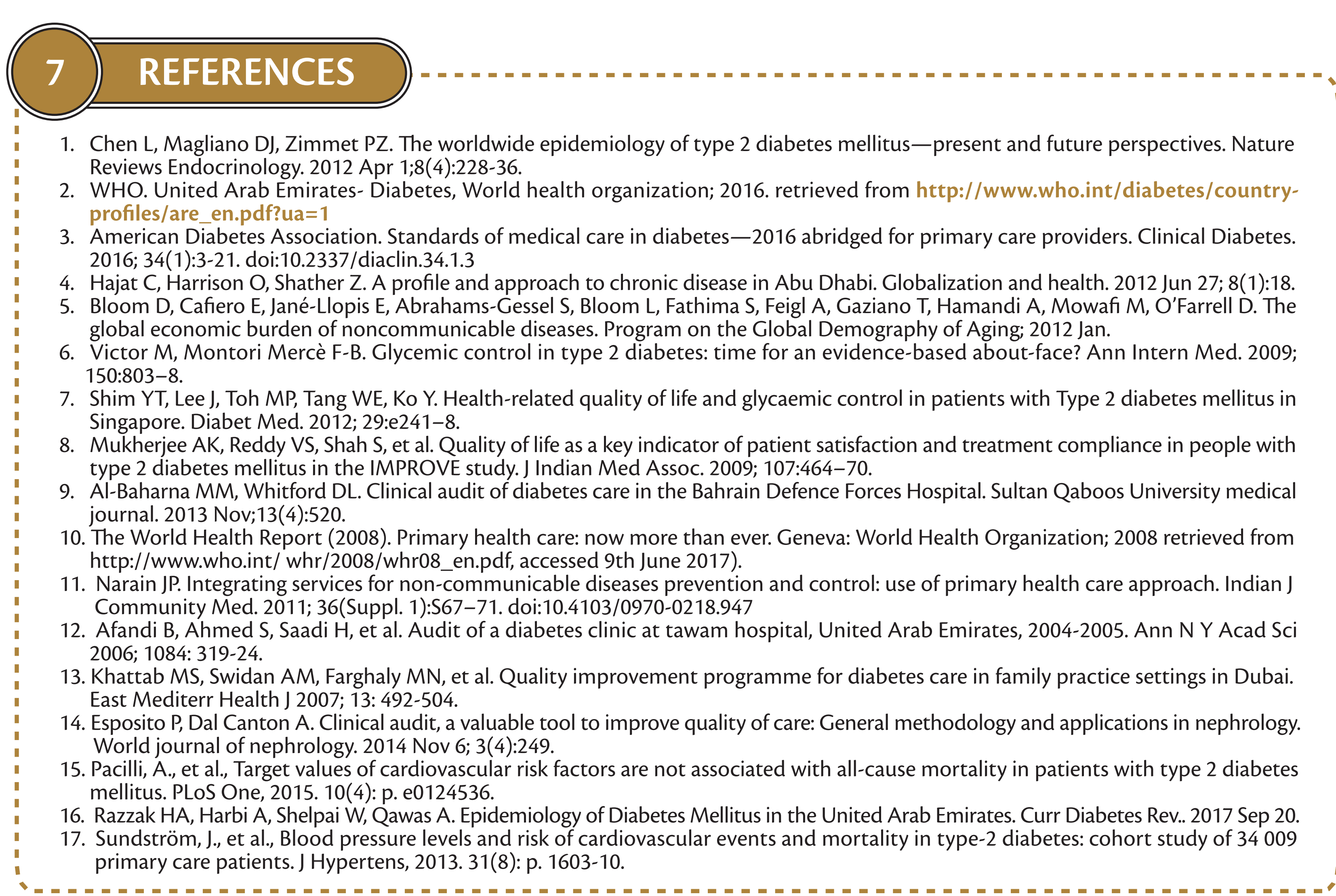

Non-communicable Diseases Section;

Primary health care department, and Statistics and Research Center. Ministry of Health and Prevention, Dubai, United Arab Emirates. 